\title{
Research on the Application of Chinese Folk Art Modeling in Graphic Design
}

\author{
Yaxiang Ding \\ Jiangsu Vocational Institute of Commerce, Jiangsu, Nanjing, 211168
}

\begin{abstract}
Folk art modeling is an independent visual symbol system and it is developed on the basis of primitive art, with the original characteristics of human thinking. Folk art is deeply influenced by folk-custom consciousness and psychological restriction. It adopts the expressive ideas of free expression, free time and space, symbolic meaning and yin-yang concept. Dynamic modeling method, decorative deformation method, geometric modeling method and text representation all have reference to be used, enriched the visual communication design language.
\end{abstract}

Keywords: folk art; modeling; graphic design

\section{Introduction}

Folk art is the ancient nation in our daily life to create, reflecting the people's basic necessities and the refraction is the desire of the people pray for good and it is the most simple working people's artistic thinking and artistic expression of the language. It is a kind of art treasury of modern art design. It is rich in shape, rich in variety, rich in connotation, has a long history, and stores precious knowledge, emotion and science and technology. On the plane design, you can use their unique artistic language, from multi-level transmission of national culture, revealing its meaning. Modern success of many graphic design works, including some by the international design community recognized, on behalf of the Chinese style of the masterpiece, the reason for their success, and based on the local, the absorption of folk art nutrition are inseparable.

Imagery comes from Chinese classical aesthetics, "meaning" is subjective thinking, "image" is an objective reality. "Imagery is beyond the existence of 
subjective and objective existence of another is the natural and human subjective spirit of unity, it is a philosophical realm, an aesthetic realm, is a kind of ideal of life."

\section{The modeling features of "artistic conception "in folk art}

"Folk art fully embodies the working people's true love for life, the pursuit of happiness, hope, the auspiciousness, justice, truth and goodness of praise, for a better life of the yearning .It's exaggerated form, art form lively, The technique of expression is simple but bold and clear, which makes the emotion of the working people manifest incisively and vividly in the folk art. It is rich in subject matter, which not only expresses the familiar things of the masses, but also reflects their rich artistic imagination., It can be said that folk art, is created by the working people themselves, the real performance and service in their lives, set aesthetic appreciation and practical function in one, full of local flavour, widely circulated in the art of the masses.

\subsection{The performance of straight expression}

The unique modeling system and artistic system of folk art show its vitality of freedom. It inherits the spirit of Chinese traditional art more and shows the important characteristics of Chinese local culture. Various forms do not rigidly adhere to the reality of objective things. They do not express certain concepts according to the true image of nature, but attach importance to the creation of subjective ideas, assumptions and assumptions in the artistic expression, and conceal the author's subjective feelings.

Emotion is a kind of psychological response to objective things. In addition to stimulating the recipient's visual nerve, attracting the attention of the recipient, strengthening memory and understanding, graphic design should stimulate the recipient's association, imagination and emotional experience. The graphic design is not only accurate and effective to convey information, but it also needs a kind of mood, there is a certain mood, so that designers form a rich association and imagination, and even produce strong emotional resonance. Thus, emotion is like a design of the main line, throughout the graphic design is always. In the design of creative thinking stage, as long as the start from the situation, from the sense of hair, can be lifeless from the image, to create a spark of consciousness of life, so that design inspire their own while infecting the audience, and ultimately to convey information the goal of.

\subsection{The performance of free time and space}

Folk art is limited by the performance materials, and should not show complex scenes and three-dimensional space. In the combination of time and space, the folk art works adopt the combination of free time and space, and the screen reflects a complete and balanced. Folk artists with their own feelings in the 
material to display their talent, the things in a level of performance, the selfunderstanding, emotion into them, operating the traditional structure of the layout of the law, for the composition of flat head or composition. Head-up composition is one of the distinctive forms of folk art in China. It is characterized by unobstructed view of the landscape and scattered parallel development. Combined composition abandon the traditional concept of perspective, completely breaking the limitations of time, space, the proportion of relations, according to the needs of the subject in a picture of this will not directly related to, at different locations, time of different characters, Of the screen segmentation or form of law, creatively and orderly combination. This combination of composition mode mainly depends on the screen image of the primary and secondary, the actual situation, balance and other forms of law to unify the screen, so that the screen has a novel, fascinating decorative effect.

Folk art advocates analyze the same object from different perspectives and from multiple perspectives, from the perceptual knowledge obtained from observation, to form a rhythmic, multi-dimensional combination of space. It brings new ideas to space design, which bring new enlightenment to the graphic design: all kinds of meaningful graphics, multi-view design thinking to create a new space-time concept, the different time and space of the organically combine to open up the design The spatial category of thought enriches the language of visual art.

\subsection{The performance of symbolic meaning}

In the traditional Chinese way of thinking and cultural system, a very important feature is the universal significance of the symbolic way of thinking and expression. In the folk art in this symbolic and symbolic function, it is omnipresent, was shown incisively and vividly. Folk art, on the basis of the ancient symbols, builds up an independent and colourful visual symbol system, which has the profound meanings of reproductive worship, ancestor worship, nature worship, totem worship, and so on. In folk art, the colourful symbolic symbol occupies the leading role. A long symbol - implied back to implement, all clear that the vortex is on behalf of the sun and light, gourd symbol of the extension of generations, the bite of coins to bite the symbol of blessing to the front of the red in China is a symbol of good fortune, which embody the symbol of folk art Characteristics of symbols.

Folk art has a rich visual and cultural resources, this traditional symbolic semantics in modern design has also been given new meaning. The design can be adapted to their design theme, borrow some symbolic meaning of symbols, and some of its changes and innovation to express a theme of emotion, so that both the traditional folk art graphic shape and charm, and Modern graphic design means. For example, China Unicom's logo is the use of the Buddhist eight treasures from the "eight auspicious" one "long" shape, whichever is "a long stream of raw and living, complementary" meaning Unicom's communications No to exhaustion, the course of time. This auspicious implication of the 
application, so that the design of the graphic less some commercial atmosphere, adding an affinity.

\subsection{The performance of the concept of yin and yang}

Yin and yang is one of the oldest philosophical concepts in China. The general outline of the Yellow Emperor's Canon of Internal Medicine is "yin and yang, the fundamental of all things," and it is the foundation of yin and yang philosophy, as well as the production of folk art. Lay the ideological and aesthetic foundation. The real part of the screen is "real" space, the empty part of the invisible space as "virtual" space, in folk art, the relationship between black and white is really exaggerated stress, "the white when black, rather empty real" speak is the actual situation Corresponding to a relationship.

In the folk paper-cut in particular attention to the picture of the virtual space blank processing, especially on the image of the contours of the ups and downs formed by the blank shape is beautiful, the overall picture of the size of the blank, density, whether the harmony is very particular about its strict structure, a high degree of summary and simple shape, it is difficult to see the screen extra space. Modern graphic design in this overall aesthetic concept under the influence of benefit, design composition should be primary and secondary clear and concise, any content irrelevant to the subject should be boldly deleted to increase the attention value of the screen, the clever use of "Blank" the design language. "Blank" is the imagination space created by designers in the creative design, in accordance with the law of the design of the screen space of anti-thinking, after careful thought, it has created the mood, provide imagination, visual ease, visual focus Multi-value function.

\section{The modeling features of "like" in Chinese folk art}

\subsection{The dynamic silhouette modeling method}

The dynamic silhouette of the modeling method of object modality characteristics is a unique folk art modeling techniques, especially in paper cutting and shadow play to represent. Silhouette modeling is based on the two-dimensional image of the outer contours of the image to represent the image, the outline of the body without the characterization, or simple engraving without decorative treatment, so it is the most attention to shape and outer contour of the United States, the intensity of the United States. Pay attention to the overall performance of the dynamic modality and choose the most characteristic point of view and modal exaggeration. Such as the characters in the picture after the omission of the five characters to the imagination of the audience space, people experience the scene scenarios connotation.

Dynamic silhouette modeling method has a high degree of simplification, flat processing characteristics, in the graphic design of the design element processing, you can fully learn from this processing approach. Such as red wine packaging, 
the use of silhouettes in the form of packaging - the image of the grandmother as the main performance, fully embodies the pure quality of the product.

\subsection{The decorative deformation method}

Folk art is "decorative plastic arts", that is, folk artists use "decorative" or "artisans of" performance techniques, not to pursue realism, and strive to reflect the subjective consciousness. "Its rich imagination, the creation of the autonomy of the various common objects in daily life subject to the needs of the decorative beauty, the reality of many concepts to return to the visual purely visual, and then selectively applied in the creation of folk art performance, In order to further enrich and enrich the theme and beautify the purpose of the work."Decorative in the eyes of folk artists is one of the important means of expression, but also reflects the different talents and literacy of each author, showing the everchanging mood and Charm.

Designers in the design creation, the design elements to image or symbolic decoration deformation processing, in accordance with the law of the United States to create, after careful observation, starting from the characteristics of grasping, summarizing the essence of the best embodiment of image, the richest performance Force the external form, retain the most important, most essential things, the rest of the part removed. This decorative simplification, derived from the subjective abstraction of the objective image, is relative to the representation of the object of the objectivity of the expression, is a form of representation of the reproducibility.

\subsection{The geometric modeling method}

The sublimation of Italian "image" in the creation of folk art graphics is also reflected in the abstract generalization of graphics. Abstraction of the objects in life is the result of the observation of natural objects. Artists will summarize, compare, analyze and abstract the inner forms of nature, while neglecting their concrete images and abstracting the concrete the result, in this abstract way, to express people in the world of all things in some form of understanding, to express people's vision and hope.

Graphic design for this abstract geometric modeling method is more widely used in the design of the visual elements of the basic, simple, breaking the original naturalized expression model, the simple abstract elements into the order of the arrangement, Looking for the structural principle of music, a variety of abstract form organically constitute the aesthetic rhythm and artistic flavour of the form, the development of the ultimate form of emotion into the rational analysis, so that all kinds of abstract form organically combined into With the aesthetic flavour of the form, so that people in the abstract field to find a new design language. 


\subsection{The text representation}

Such as the most common is to Kyrgyzstan Chinese characters as the theme of the hi flower paper-cut, with "hi", "Double Happiness" is the most common word or "year after year", "happy" and other Chinese characters and flowers, birds and animals And often with a beautiful vocabulary and graphics and use, such as "spend a good full moon, dragon and phoenix, and all the best" and peony, peach and other flower-shaped combination of intuitive image to express the attitude of people's attitudes towards life. Text is an indispensable factor in distinguishing commodity identification systems. In the creation of packaging design, the brand name, trademark, composition, use, instructions, manufacturers need to use words to express the text in the packaging design occupies an important position. It is also constitute an integral whole of the screen an important part of the composition of the appearance of the United States constitute the packaging of the key. Form of the United States and the text of the product design to add luster to the packaging, so that consumers feast for the eyes, and the image and the goods linked together on the packaging of goods has a better idea.

\section{Conclusion}

Folk art is one of the mother cultures of our nation, it is the spiritual pillar of our art, the mother of art creation and the nutrition source for Chinese civilization. As design talent in new era, we should grasp the law of modern design, make our mother culture and Western design ideas better integration and recycling, so the design of the Chinese charm shine in the world design stage.

\section{References}

[1] Xu Xiaogeng. Modern Design Art. Chongqing: Yangtze River Literature and Art Publishing House, pp.68-70, 2005

[2] Fan Tao. The Unique Authenticity of Folk Art Modeling Language, 8 (5), pp.87-91, 2002 